\title{
AVALIAÇÃO DA VALIDADE E CONFIABILIDADE DO GLOBAL ENTREPRENEURSHIP INDEX
}

Eduardo Dionisio ${ }^{1}$

Edmundo Inácio Júnior ${ }^{1}$

Dirceu Da Silva ${ }^{1}$

${ }^{1}$ Universidade Estadual de Campinas 


\section{AVALIAÇÃO DA VALIDADE E CONFIABILIDADE DO GLOBAL ENTREPRENEURSHIP INDEX}

Resumo: O Global Entrepreneurship Index (GEI) fornece um conjunto de informações e análises comparativas sobre o perfil empreendedor dos países. No entanto, ainda que o GEI se baseie nas recomendações da OCDE sobre construção de indicadores compostos (índices), não identificamos nenhum estudo (nem mesmo elaborado pelos próprios desenvolvedores do índice) sobre a validade, confiabilidade e acurácia desses indicadores para mensurar os aspectos multifacetados do empreendedorismo. Tendo em vista essa lacuna, nessa pesquisa, avaliamos a validade, confiabilidade precisão do GEI, por meio da aplicação da modelagem de equações estruturais. Os resultados revelam que o GEI é adequado e significativamente preciso para avaliar o empreendedorismo em nível de país. No entanto, identificamos que o componente do índice que capta a percepção da população sobre oportunidades. Por outro lado, também identificamos que o componente não capta aspectos tecnológicos e de mercado associadas a percepção de oportunidades empreendedoras.

Palavras-chave: Structural Equation Modeling. Ecossistema de empreendedorismo. Índice composto. Indicadores.

\section{$1 \quad$ Introdução}

O empreendedorismo é um fenômeno socioeconômico que impulsiona níveis agregados de inovação, empregos, competitividade e crescimento econômico (ÁCS; AUDRETSCH; LEHMANN, 2013; LAFUENTE; SZERB; ÁCS, 2015; TASNIM; AFZAL, 2018). O empreendedorismo pode contribuir para o desenvolvimento socioeconômico local e/ou regional (STANGLER; BELL-MASTERSON, 2015). Dada as contribuições, o empreendedorismo é cada vez mais reconhecido pelos governos como uma atividade que deve ser incentivada (HART, 2003; LINK; SCOTT, 2010; RIBEIRO-SORIANO; MARTÍN-GALINDO, 2012; SMALLBONE, 2016; GIRAUDO; GIUDICI; GRILLI, 2019).

No entanto, o desempenho (inovação) e impactos da atividade empreendedora pode variar entre países, regiões, cidades e entre setores (AUTIO et al., 2014). O empreendedorismo é um processo condicionado por vários fatores e, ainda que seja um resultado do comportamento, motivações e competências individuais, depende do reconhecimento e exploração de oportunidades, disponibilidade de recursos e das condições do ambiente em que os empreendedores operam (RADOSEVIC; YORUK, 2013), isto é, infraestruturas, e instituições locais e regionais - sociais, econômicas e financeiras (STAM, 2015).

Para formular políticas de apoio e ações para incentivar o empreendedorismo, é fundamental avaliar comparativamente regiões e países com subsídio de indicadores adequados para mensurar o fenômeno empreendedor (SHANE, 2009). No entanto, mensurar o empreendedorismo em nível nacional é um desafio, pois não há consenso sobre o que constitui um conjunto coerente de indicadores (OECD, 1998).

A construção de indicadores tem funcionado até recentemente em três abordagens quase independentes, que incluem: estatísticas de entrada de empresas (indicadores de produto); análise do comportamento e das atitudes empreendedoras, incluindo valores e preferências da população adulta (indicadores de atitudes); e a avaliação do contexto ou das condições para 
empreender - indicadores de contexto (ÁCS; AUTIO; SZERB, 2014). Ainda que o empreendedorismo seja reconhecido como um fenômeno multifacetado, as principais abordagens tratam de aspectos diferentes do empreendedorismo, portanto, nenhuma é capaz de fornecer um quadro completo do estado do empreendedorismo (CODURAS; AUTIO, 2013).

De fato, se consideramos o empreendedorismo como a disposição para operacionalizar um negócio com base no reconhecimento de uma oportunidade e utilizando os recursos disponíveis, não devemos limitar a medida de empreendedorismo à firma e ao emprego, pois isso resultará em uma visão reduzida sobre empreendedorismo em uma região ou país (ÁCS; AUTIO; SZERB, 2014). Assim, uma das tendências mais recentes dos estudos em empreendedorismo é unir as três abordagens de indicadores para criar índices integrados de empreendedorismo (CODURAS; AUTIO, 2013).

Esses índices representam uma visão ampla do empreendedorismo baseada na literatura dos ecossistemas empreendedores (EE) que enfatiza o papel do contexto (fatores institucionais, sociais e de estrutura) no impulso e inibição do empreendedorismo. O Global Entrepreneurship Index (GEI) formulado por Ács e Szerb (2009), é o primeiro índice integrado, baseado nas informações fornecidas por diversas pesquisas de empreendedorismo como o Global Entrepreneurship Monitor (GEM), para o comportamento empreendedor e estatísticas e características da atividade empreendedora; e Ease of Doing Business (EDB), Global Competitiveness Index (GCI), Economic Freedom Index (EFI), Corruption Perception Index (CPI) e Economic Complexity Index (ECI) para avaliar o contexto e condições para empreender. O GEI é composto por 14 indicadores subdivididos em 3 subíndices que fornecem um quadro sobre o comportamento empreendedor da população adulta, as características dos empreendedores e seus impactos socioeconômicos.

Desde 2011, pesquisadores associados ao GEI publicam anualmente estudos de empreendedorismo a nível de país e o índice tem sido utilizado como marco analítico para compreender o empreendedorismo sob uma perspectiva sistêmica em países e/ou regiões (por exemplo, ÁCS et al., 2014; SZERB et al., 2014; ATIASE; MAHMOOD; BOTCHIE, 2018; SZERB; TRUMBULL, 2018; THU HA; BA HOA, 2018). No entanto, ainda não existem estudos sobre a validade, confiabilidade e acurácia dessa metodologia para mensurar o empreendedorismo. As tentativas para mostrar a validade dessa metodologia se restringem a matrizes de correlação entre indicadores que compõe o GEI e testes de correlação entre GEI, DBI, GCI, CPI e PIB (ver ÁCS; SZERB, 2009).

Tais testes revelam que os indicadores se correlacionam, mas não indicam sua acurácia e confiabilidade tanto de indicadores quanto dos subíndices para avaliar o empreendedorismo. Técnicas estatísticas mais sofisticadas, como a modelagem de equações estruturais (MEE) permitem identificar se um índice de empreendedorismo é válido como método para explicar um fenômeno, pois a MEE fornece informações que explicam relações entre múltiplas variáveis (HAIR et al., 2016).

Diante do exposto, questionamos se os indicadores e subíndices do Global Entrepreneurship Index são confiáveis e precisos para mensurar o empreendedorismo em nível de país?

Para tanto, essa pesquisa avalia a validade, confiabilidade e precisão do Global Entrepreneurship Index, por meio da aplicação da técnica de modelagem de equações estruturais, utilizando o software Smart-PLS. 
Esse artigo está estruturado em 5 seções. Além dessa introdução, desenvolvemos uma revisão da literatura sobre os ecossistemas de empreendedorismo e apresentamos uma síntese do Global Entrepreneurship Index. A seção 3 descreve os procedimentos metodológicos. Os resultados e discussões são apresentados na seção 4 e as considerações finais e considerações para trabalhos futuros podem ser encontradas na seção 5 .

\section{$2 \quad$ Ecossistemas de empreendedorismo}

O conceito de ecossistemas de empreendedorismo (EE) deriva do termo "ecossistemas de negócios" de Moore (1993), o qual se baseia em uma interpretação biológica para explicar a organização de uma indústria ou cadeia de valor centralizada. No entanto, os ecossistemas empreendedores se caracterizam pela descentralização e interação interdependente de seus atores, que incluem: instituições formais e não formais, organizações e serviços de apoio, infraestrutura física, universidades, entre outros (STAM, 2015).

No entanto, o termo se popularizou entre acadêmicos, pesquisadores, formuladores de políticas, empreendedores e entusiastas, devido ao sucesso comercial do livro de Feld (2012) intitulado "Startup communities: Building and entrepreneurial ecosystem in your city" e do trabalho de Isenberg (2010) "How to start an entrepreneurial revolution" publicado pela Harvard Business Review. Esses autores contribuíram para a difusão da noção de que um conjunto de atores e fatores locais interferem no desempenho da atividade empreendedora.

Apesar da popularidade do termo ecossistema empreendedor, ainda não há uma definição amplamente compartilhada pela literatura (STAM, 2015). O conceito de EE pode ser visto como "um guarda chuva conceitual que abrange uma variedade de perspectivas diferente sobre a geografia do empreendedorismo, em vez de uma teoria coerente" (SPIGEL, 2017, p. 1). As primeiras definições de ecossistemas de empreendedorismo se concentram na complexidade das interações entre atores e componentes e, na criação de novos empreendimentos, esse aspecto é visto como o objetivo final de um ecossistema (ver VAN DE VEN, 1993; SPILLING, 1996; NECK et al., 2004; COHEN, 2006). A segunda geração de estudos se concentrou em descrever as estruturas, atores e componentes dos EE, assim como enfatizar o papel dos ecossistemas para desenvolvimento regional (ISENBERG, 2011; FELD, 2012; WEF, 2013; MASON; BROWN, 2014).

A ascensão de estudos sobre os elementos constituintes dos EE também encontrou críticas. De acordo com vários autores (por exemplo, STAM, 2015), esses estudos se concentraram em descrever os elementos que constituem um ecossistema, enquanto as conexões entre esses elementos e sua evolução foram negligenciadas (ÁCS et al., 2016). Ao mesmo tempo a pressa por mapear e descrever os elementos que constituem os EE, apenas forneceu uma "longa lista de fatores relevantes sem um raciocínio claro de causa e efeito" (STAM, 2015, p. 1764). Esses estudos partem de uma perspectiva estática dos EE, sem considerar a dinâmica de sua evolução (ALVEDALEN; BOSCHMA, 2017), onde a todos os elementos é atribuído o mesmo grau de importância (STAM, 2015).

Com base nas contribuições e lacunas dos estudos anteriores, Stam (2015) define ecossistemas de empreendedorismo como "um conjunto de atores e fatores interdependentes coordenador de maneira a permitir o empreendedorismo produtivo dentro de um território específico" (p. 5). Esse autor contribuiu para a mudança do lócus da investigação de ecossistema 
para o empreendedorismo produtivo, qualquer atividade econômica inovadora, orientada para o crescimento e que cria valor socioeconômico para a sociedade (BAUMOL, 1993).

Os formuladores do Global Entrepreneurship Index (ÁCS; SZERB, 2009; ÁCS; AUTIO; SZERB, 2014) incorporaram a ideia de ecossistema empreendedor e a dimensão de empreendedorismo produtivo em seu índice, enfatizando que a interação entre indivíduos (empreendedores e potenciais empreendedores) e seu contexto institucional impulsiona o reconhecimento e exploração de oportunidades, por meio de um processo de alocação de recursos que resulta na criação e operacionalização de novos negócios potencialmente inovadores; e orientados ao crescimento e internacionalização.

\subsection{Global Entrepreneurship Index (GEI)}

Em 2008, Zoltán J. Ács e Laszló Szerb, pesquisadores membros da equipe húngara do consórcio Global Entrepreneurship Monitor, iniciaram um projeto de pesquisa (que resultou no índice GEI) com vistas a formular implicações políticas a partir da combinação entre dados do GEM e de pesquisas que avaliam o contexto empreendedor (como Ease of Doing Business, Global Competitiveness Index, Economic Freedom Index, entre outras). Ács e Szerb visavam preencher as lacunas dos estudos de empreendedorismo do GEM, isto é, examinar os efeitos combinados entre contexto e atividade empreendedora e, por fim, fornecer diagnósticos sobre as forças e fraquezas dos ecossistemas empreendedores em nível de país, auxiliando na formulação de políticas de empreendedorismo mais adequadas a cada país (SZERB, 2013).

O GEI fornece análises comparativas sobre o desempenho dos ecossistemas empreendedores a nível de país, por meio de um conjunto de indicadores (pilares) agregados em três subíndices: Entrepreneurial Attitudes Sub-Index (ATT); Entrepreneurial Abilities SubIndex (ABT); Entrepreneurial Aspirations Sub-Index (ASP). A média aritmética desses subíndices determina o desempenho do ecossistema empreendedor nacional e a classificação dos países.

Cada subíndice agrega um conjunto de pilares (indicadores), no total o índice é estruturado em 14 pilares, cada um deles é composto por uma variável individual e uma institucional, correspondentes aos níveis micro e macro do empreendedorismo.

As variáveis individuais descrevem as atitudes da população em relação ao empreendedorismo e as características da atividade empreendedora. As variáveis institucionais descrevem as características do contexto empreendedor, como dinâmica do mercado, eficiência de um país para implementar políticas públicas, capacidade das empresas locais de absorver as tecnologias mais recentes e de perseguir estratégias de diferenciação. Dessa maneira, cada um dos 14 indicadores é o resultado da combinação entre uma variável individual e outra institucional (ÁCS; SZERB; LLOYD, 2018).

As variáveis individuais são disponibilizas ao público pelo GEM, assim como as variáveis institucionais, no entanto, essas são de responsabilidade de organizações internacionais, como o Fórum Econômico Mundial, Heritage Foundation, Banco Mundial, UNESCO, entre outras.

A Tabela 1 apresenta uma breve descrição dos pilares e variáveis que compõem cada subíndice do GEI. 
Tabela 1 - Estrutura do Global Entrepreneurship Index

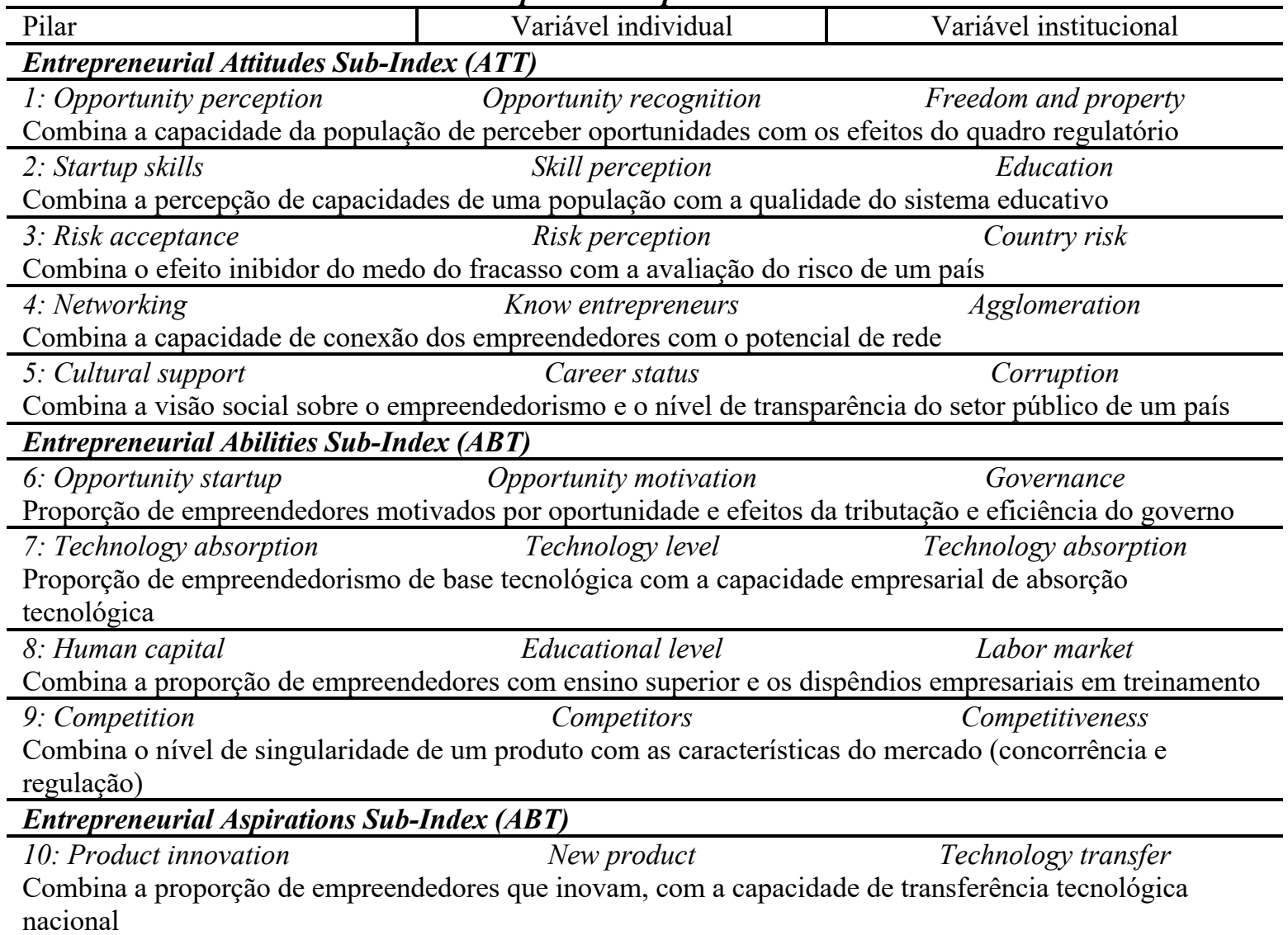

11: Process innovation New technology Science

Proporção de empreendedores que inovam em processos produtivos e a qualidade do sistema de inovação

12: High growth Gazelle Finance and strategy

Empreendedores de alto crescimento e capacidade das empresas de adotar estratégias de diferenciação

13: Internationalization Export Economic complexity

Combina empreendedores que exportam com o nível de complexidade econômica de um país
14: Risk capital
Informal investment
Depth of capital market

Combina a proporção de financiamento informal e a profundidade do mercado de capitais de uma economia

Fonte: Elaborado a partir de Ács, Szerb e Lloyd (2018)

Visando facilitar a compreensão dos pilares, subíndices e variáveis, adotou-se a nomenclatura original, no idioma inglês.

\section{$3 \quad$ Método de pesquisa}

\subsection{Dados e amostra}

Para avaliar a precisão do GEI, utilizamos dados do próprio índice, publicamente disponíveis no site (http://www.thegedi.org/) da organização denominada The Global Entrepreneurship and Development Institute (GEDI), a qual é responsável pelos estudos de empreendedorismo GEI. 
Nossa amostra é composta por 137 países. Em virtude da restrição de páginas, optou-se por mencionar apenas a quantidade de países mensurados pelo GEI, os quais estão disponíveis no livro The Global Entrepreneurship Index 2018 (ÁCS; SZERB; LLOYDE, 2018).

Para cada um desses países, coletamos dados referentes aos 14 pilares de empreendedorismo do GEI (opportunity perception, startup skills, risk acceptance, networking, cultural support, opportunity startup, technology absorption, human capital, competition, product innovation, process innovation, high growth, internationalization e risk capital) referentes ao relatório de 2018.

A fim de facilitar a visualização dos pilares e sua rápida associação ao seu respectivo sub-índice, criamos uma nomenclatura para cada pilar baseada em seu subíndice correspondente (Tabela 2).

Tabela 2 - Abreviação dos pilares

\begin{tabular}{|c|c|c|c|c|}
\hline \multicolumn{2}{|c|}{ Abreviação/pilares ATT } & Abreviação/pilares ABT & \multicolumn{2}{|c|}{ Abreviação/pilares ASP } \\
\hline ATT_1 & Opportunity perception & Opportunity startup & ASP_1 & Product innovation \\
\hline ATT_2 & Startup skills & Technology absorption & ASP_2 & ss innovation \\
\hline ATT_3 & Risk acceptance & Human capital & ASP_3 & High growth \\
\hline $\mathrm{ATT}_{-} 4$ & Networking & $\mathrm{ABT}_{-}^{-} 4$ Competition & $\mathrm{ASP}_{-}^{-} 4$ & Internationalization \\
\hline ATT_5 & Cultural support & & ASP_5 & Risk capital \\
\hline
\end{tabular}

Fonte: Elaboração própria

Por exemplo, o pilar opportunity perception foi denominado ATT_1 pois faz parte do subíndice ATT e é o primeiro indicador desse conjunto. O pilar human capital foi denominado ABT_3 pois compõe o subíndice ABT e é o terceiro componente desse conjunto.

\subsection{Modelagem de equações estruturais}

Para alcançar o objetivo de pesquisa, foi aplicado um tratamento estatístico multivariado de dados, por meio da Modelagem de Equações Estruturais (MEE). De acordo com Hair et al. (2016) a MEE é adequada para avaliar o quanto um modelo é capaz de explicar os aspectos multifacetados de um fenômeno.

Ringle, Silva e Bido (2014) incentivam o uso do software Smart-PLS pela possibilidade de modelar variáveis latentes com indicadores formativos (formam o constructo e não tem correlação entre si), em vez de indicadores reflexivos (são formados por constructos) em modelos estruturais.

Para Gudergan et al. (2008), o Smart-PLS é adequado para analisar a adequabilidade de modelos, pois organiza internamente uma Análise Fatorial Exploratória (AFE) para que se obtenha um conjunto de variáveis representativas dos constructos. O software também organiza uma Análise Fatorial Confirmatória (AFC) para avaliar o quanto cada indicador se ajusta a um modelo. De acordo com Hair et al. (2014) dentre as justificativas para o uso do Smart-PLS se destaca sua capacidade de fazer a transição entre as técnicas de AFE e AFC.

O primeiro passo para aplicar a modelagem de equações estruturais no GEI é construir um diagrama de caminhos (path diagram) de relações causais que represente os três subindices do empreendedorismo (ATT, ABT e ASP), denominados "constructos" ou "variáveis latentes" (VL) pela linguagem da literatura de análise multivariada de dados. Com base na edição de 2018 do GEI (ÁCS; SZERB; LLOYD, 2018), construirmos o diagrama de caminhos, por meio 
de equações estruturais, ligando os constructos/variáveis latentes (sinalizadas por elipses) as variáveis observáveis (VO) ou indicadores (sinalizados por retângulos), conforme ilustrado na Figura 1.

\section{Figura 1 - Diagrama de caminhos}

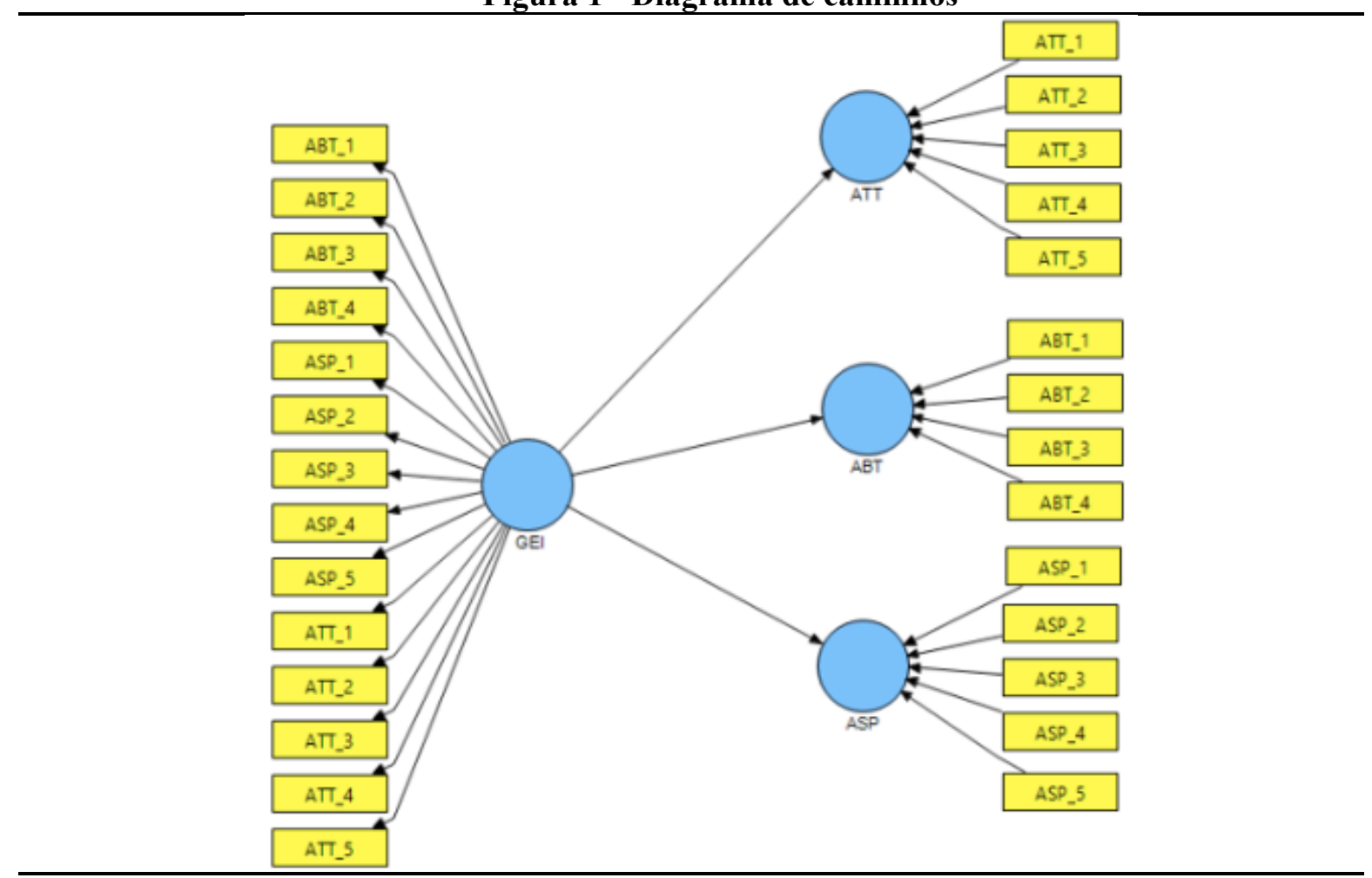

Fonte: Elaboração própria

Como se trata de um modelo formativo, foi criado um constructo de segunda denominado GEI, que agrega os 14 pilares dos constructos de primeira ordem (ATT, ABT e ASP). Criar um constructo de segunda ordem é um recurso matemático para avaliar as relações entre as VL de primeira ordem com um modelo mais genérico.

Como no modelo há três constructos formativos, fez-se a avaliação da multicolinearidade. Dessa forma, foram calculados os valores do VIF (variance inflation factors) pelo uso do software SPSS. Os valores resultantes para todos os pilares mensurados e presentes nos constructos formativos (ATT, ABT e ASP), ficaram abaixo de 10 (variando de 1,88 a 7,42), sugerindo que se pode desprezar a presença do fenômeno avaliado (KUTNER et al., 2004).

\section{$4 \quad$ Resultados e discussões}

\subsection{Análise descritiva dos resultados}

Após a modelagem de equações estruturais do GEI utilizando o software Smart-PLS, o passo seguinte foi o cálculo do modelo, exibido na Figura 2. 


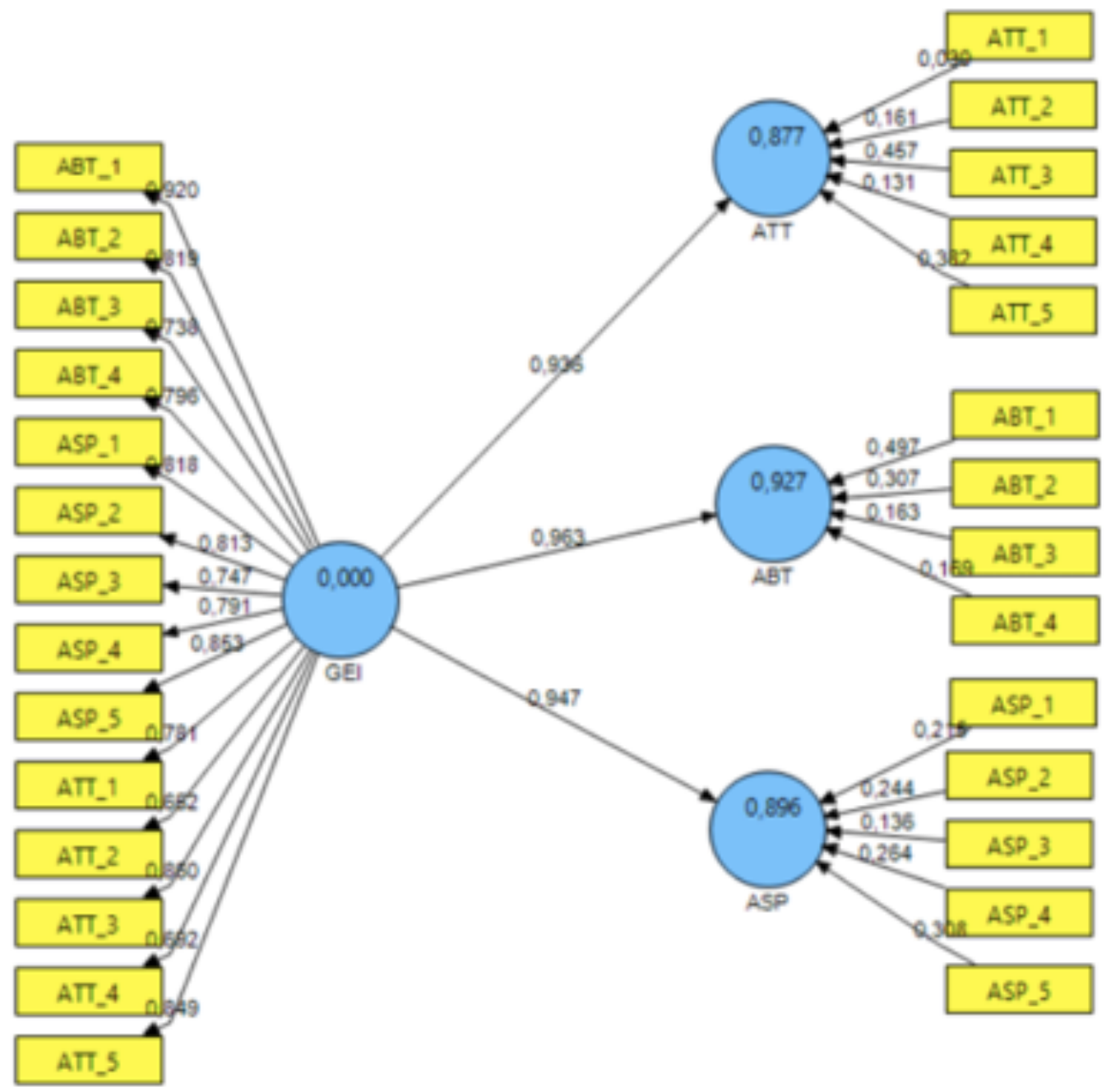

Fonte: Elaboração própria

A Figura 2 apresenta uma esquematização do modelo GEI contendo as cargas fatorais para cada constructo e seus respectivos indicadores: ATT $(0,877)$, ATT_1 $(0,039)$, ATT_2 $(0,161)$, ATT_3 $(0,457)$, ATT_4 $(0,131)$, ATT_5 $(0,382)$, ABT $(0,927)$, ABT_1 $(0,49 \overline{7})$, ABT_2 (0,307), ABT_3 (0,163), ABT_4 (0,159), ASP $(0,896)$, ASP_1 $(0,215)$, ASP_$_{-} 2(0,244)$, ASP_3 $(0,136)$, ASP_4 $_{(0,264)}$ e ASP_5 $(0,308)$. Embora a Figura 2 apresente um constructo denominado GEI com valor 0 para cargas fatoriais, trata-se de um constructo de segunda ordem, utilizado para representar modelos formativos, portanto, avaliações de cargas fatoriais não se aplicam (HAIR et al., 2014).

Após o cálculo das cargas fatoriais, avaliamos o coeficiente de determinação $\left(\mathrm{R}^{2}\right)$, uma medida descritiva da qualidade do ajuste obtido, que basicamente, explica, por meio de regressões, o quanto o modelo foi capaz de explicar os dados coletados. 
$\mathrm{O} \mathrm{R}^{2}$ varia entre 0 e 1 , portanto, quanto mais próximo a 1 , maior será a validade da regressão e consequentemente, o modelo será considerado ajustado. Por outro lado, o valor de $\mathrm{R}^{2}$ tende a crescer quando o número de observações é menor. Os três constructos apresentaram coeficientes de determinação próximos a 1 , com destaque para o constructo $\mathrm{ABT}$, cujo $\mathrm{R}^{2}$ é de 0,927, seguido por ASP $(0,896)$ e ATT $(0,877)$. Esses valores significativamente levados indicam que o modelo é muito satisfatório.

Além da avaliação de cargas fatoriais e do coeficiente de determinação, Hair et al. (2014) sugerem a aplicação do módulo "Boostrapping" (técnica de reamostragem), em que subamostras são sorteadas de forma aleatória, incluindo a substituição do conjunto inicial de dados. Cada uma das subamostras é utilizada nas avaliações do modelo. Esse procedimento ocorre até que se crie um conjunto significativo de subamostras aleatórias, possibilitando identificar se os indicadores (pilares) se ajustam ao modelo. Caso um indicador apresente valor igual ou superior ao valor de referência t de Student 1,96 é considerado adequada ao modelo, por outro lado, se inferior, o indicador é considerado inadequado ao modelo (Figura 3).

Figura 3 - Resultados do teste t de Student

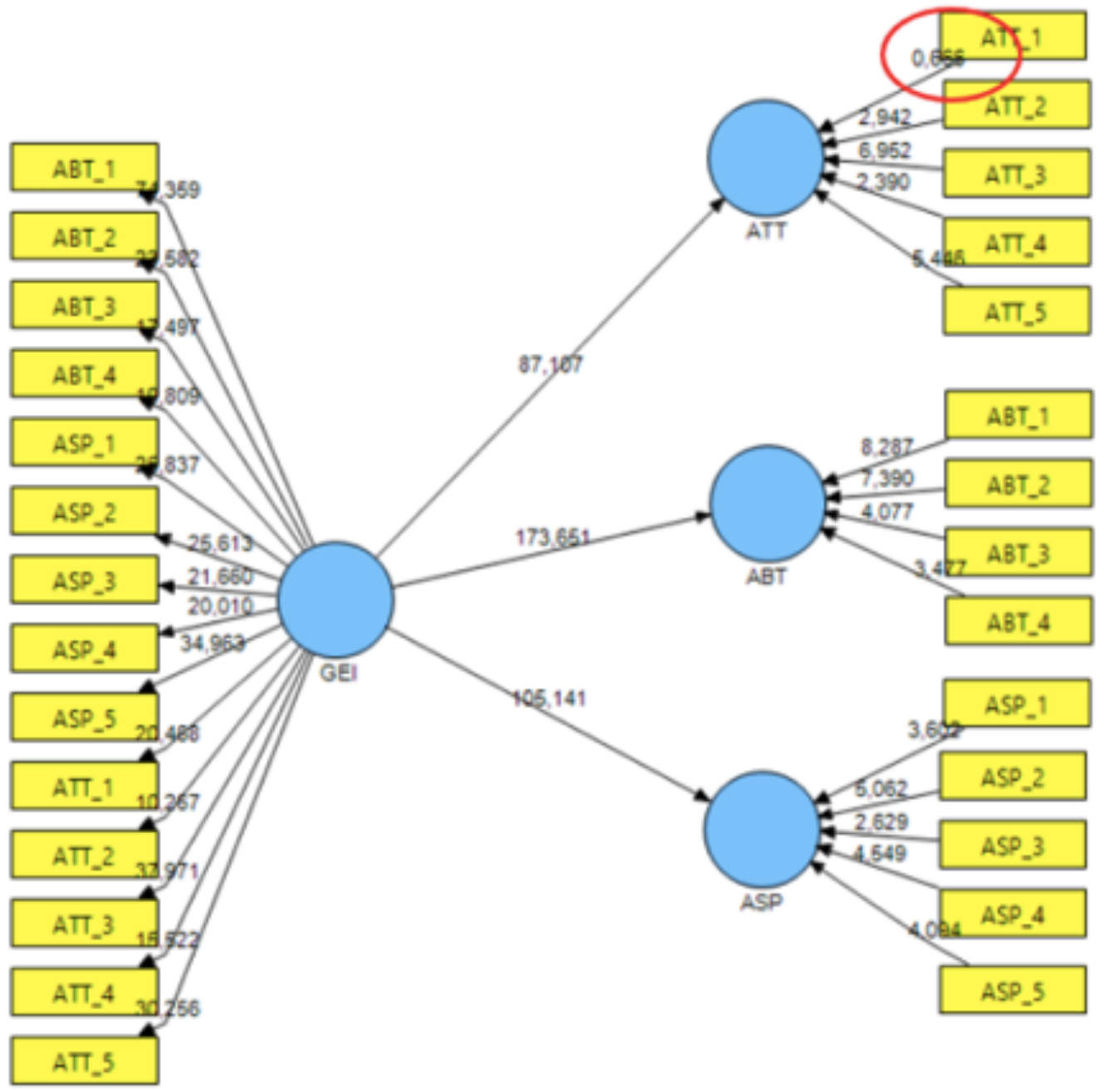

Fonte: Elaboração própria 
Todos indicadores apresentam valor de referência acima de 1,96, indicando que são significativamente ajustados ao modelo. A exceção foi oi indicador opportunity perception (ATT_1), cujo valor é equivalente a 0,66 , significativamente inferior ao valor de referência $t$ de Student $\geq 1,96$.

Após aplicar o módulo 12 e obter os valores t de Student para cada um dos indicadores. A próxima etapa recomendada por Hair et al. (2014) é avaliar a qualidade de ajuste do modelo, por meio dos testes de validade preditiva $\left(\mathrm{Q}^{2}\right.$ ou indicador de Stone-Geisser) e tamanho do efeito ( $\mathrm{F}^{2}$ ou indicador de Cohen).

O teste de validade preditiva avalia em que medida o modelo se aproxima do que se esperava dele, isto é, se o modelo tem acurácia para mensurar o fenômeno. Um modelo prefeito teria valor $\mathrm{Q}^{2}$ equivalente a 1 , indicando que um constructo retrata um fenômeno com extrema precisão. O tamanho do efeito mensura o quanto um constructo é "útil" para o ajuste do modelo. $\mathrm{O}$ valor $\mathrm{F}^{2}$ é obtido por meio de análises de sensibilidade (exclusão e inclusão de constructos), sendo 0,$02 ; 0,15$ e 0,35 considerados pequenos, médios e grandes. Dessa maneira, quanto maior o valor $\mathrm{F}^{2}$ de um constructo, maior sua relevância para o modelo (HAIR et al., 2014).

Os valores para a validade preditiva e tamanho do efeito para cada um dos constructos, incluindo o constructo de segunda ordem (GEI) são exibidos na Tabela 3.

Tabela 3 - Valores para validade preditiva e tamanho do efeito

\begin{tabular}{l|c|c}
\hline Constructos & Validade preditiva $\left(\mathrm{Q}^{2}\right)$ & Tamanho do efeito $\left(\mathrm{F}^{2}\right)$ \\
\hline ATT & 0,591416 & 0,541909 \\
ABT & 0,658830 & 0,554301 \\
ASP & 0,648552 & 0,587047 \\
GEI & 0,578712 & 0,578712 \\
\hline
\end{tabular}

Fonte: Elaboração própria

Os constructos ATT $(0,591)$, ABT $(0,659)$, ASP $(0,648)$ e GEI $(0,579)$ apresentam valores de validade preditiva superiores a 0,50 , o que sugere que todos estão significativamente ajustados ao modelo final. No que se refere ao tamanho do efeito, podemos observar uma situação semelhante, onde os constructos ATT $(0,542)$, ABT $(0,554)$, ASP $(0,587)$ e GEI $(0,579)$ por apresentarem valores de referência $F^{2}$ muito superiores a 0,35 , são considerados relevantes para o modelo final.

Embora a variável ATT_1 (opportunity perception) apresente valor inferior a t 1,96, não se ajustando ao modelo, os indicadores de validade preditiva e de tamanho do efeito indicam que os constructos estão ajustados ao modelo e são adequados para captar as propriedades dos ecossistemas empreendedores.

\subsection{Discussões}

Os resultados da modelagem de equações estruturais, mostraram que o índice GEI é robusto e significativamente preciso para avaliar os ecossistemas empreendedores em nível nacional. No entanto, identificamos que o pilar opportunity perception (ATT_1) apresentou valor equivalente a 0,66 , significativamente inferior ao valor adequado para os padrões do teste t de Student $(1,96)$. 
Esse pilar indica se a população de um país é capaz de identificar oportunidades para iniciar um negócio e o ambiente institucional torna possível explorar essas oportunidades. Trata-se de uma medida combinada de duas variáveis: opportunity recognition e freedom and property. Os dados para a primeira variável são coletados pela iniciativa Global Entrepreneurship Monitor (GEM) e mensuram a proporção da população adulta (de 18 a 64 anos) que pode identificar oportunidades para iniciar um negócio dentro de seis meses na área em que reside. No entanto, o valor dessas oportunidades depende do tamanho do mercado (ÁCS; SZERB; LLOYD, 2018). A segunda variável, freedom and property, se trata de um componente do Economic Freedom Index (EFI), que captura os efeitos do ambiente regulatório na atividade empreendedora e em negócios operacionais, assim como nos direitos a propriedade privada, isto é, se o Estado garante que a população possa obter retornos da exploração de oportunidades empreendedoras.

As explicações para esse pilar não atingir o valor de referência podem ser atribuídas tanto a heterogeneidade dos países no que se refere a proporção da população capaz de identificar oportunidades e a qualidade do quadro regulatório, quanto a restrição do número de métricas empregadas para capturar esse fenômeno.

Dos 137 países que compõe o banco de dados do Global Entrepreneurship Index, apenas 34 possuem desempenho igual ou superior a 0,50 no pilar opportunity perception (os valores para os indicadores do GEI variam de 0 a 1) sendo a maioria deles, países desenvolvidos. Os demais 103 países apresentam pontuação inferior a 0,50, fato que impacta nos resultados da modelagem de equações estruturais, mostrando que o indicador não se ajusta ao modelo.

Com vistas a identificar as razões que levam muitos países da amostra a possuirem desempenho tão baixo no pilar opportunity perception, analisamos os bancos de dados do GEM e EFI.

Os dados do GEM (2018) revelam que dos 49 países analisados, apenas 12 estão no terceiro quartil, pois apresentam as maiores proporções da população adulta que identifica oportunidades para iniciar um negócio, isto é, proporção superior a 56,11 são eles: Suécia (81,56\%), Arábia Saudita (76,3\%), Angola (74,02\%), Sudão (70,98\%), Estados Unidos $(69,83 \%)$, Polônia $(68,48 \%)$, Holanda $(66,73 \%)$, Emirados Árabes Unidos $(66,50 \%)$, Canadá (62,98\%), Chile (61,78\%), Colômbia (57,48\%) e Israel (56,23\%).

No que se refere a outra variável que compõe o pilar opportunity perception, freedom and property. Ao observar o banco de dados do Economic Freedom Index, identificamos que dos 186 países que compõe o índice, cerca de 46 estão no terceiro quartil, pois apresentam desempenho acima de 75,5 pontos na variável business freedom do EFI. No mesmo quartil se encontram cerca de 47 países, cuja pontuação na variável property rights é superior a 66,8, sendo a maioria deles, países desenvolvidos, por exemplo: Suécia, Reino Unido, Finlândia, Holanda, Irlanda, Canadá, Noruega, Coreia do Sul, Estados Unidos, entre outros.

Ao observar os bancos de dados do GEM e EFI, especificadamente para as variáveis opportunity recognition e economic freedom (business freedom e property rights) observamos que os países que apresentam as maiores pontuações na variável do GEM, são aqueles que também se destacam nas variáveis do EFI. Dessa maneira, podemos notar uma associação entre reconhecimento de oportunidades e qualidade do quadro regulatório. Como no caso da Suécia, que apresenta qualidade no quadro regulatório (desempenho significativo nos componentes 
property right e business freedom (92,6 e 89,3$)$ ) e cerca de $81,56 \%$ da população da população que reconhece boas oportunidades para iniciar um negócio.

Essas observações corroboram com afirmação de Ács, Szerb e Lloyd (2018) de que a capacidade de identificar e explorar oportunidades depende do tamanho do mercado e os retornos da sua exploração dependem da qualidade do quadro regulatório.

Dessa maneira, o pilar opportunity perception apresenta valor de referência inferior a $t$ 1,96 devido ao baixo número de países com desempenho significativo. Como quase quase todos os países com valores próximos a 1 nesse pilar são desenvolvidos, questionamos se esse indicador seria adequado para avaliar contextos econômicos heterogêneos.

O uso do componente freedom and property é recente, data de 2017, período em que o modelo conceitual do GEI foi reorganizado, anteriormente essa medida estava vinculada ao pilar opportunity motivation e o outro componente do pilar opportunity perception era um proxy da aglomeração de mercado mensurado pelo tamanho do mercado interno (medida combinada entre PIB e do saldo da balança comercial) e taxa de urbanização. Quando combinados, esses componentes permitem multiplicar o tamanho do mercado interno (potencial de demanda) pela proporção da população em áreas urbanas (ÁCS; SZERB; AUTIO, 2016).

Embora o quadro regulatório e os direitos a propriedade privada garantam liberdade para a exploração e a coleta dos recursos vindos de oportunidades empreendedoras. Alguns acadêmicos sugerem que indivíduos localizados em contextos mais intensivos em conhecimento são mais propícios a identificar e explorar oportunidades empreendedoras (ÁCS et al., 2009), isso se dá, devido ao estoque de capital humano qualificado com conhecimento prévio sobre as novas tecnologias (QIAN; ÁCS, 2013). Dessa maneira, uma população educada é uma precondição necessária para o reconhecimento e exploração de oportunidades empreendedoras (AUDRETSCH et al., 2012).

Ao avaliar a propensão empreendedora por meio da criação de um indicador composto Radosevic e Yoruk (2013) comprovaram estatisticamente que o empreendedorismo embora seja orientado para o mercado é afetado pela interação entre mercado com oportunidades tecnológicas e institucionais.

O IC proposto por Radosevic e Yoruk (2013), apresenta uma visão ampla dos fatores que afetam o reconhecimento de oportunidades empreendedoras, que contemplam dimensões institucionais, tecnológicas e de mercado. A dimensão institucional trata do quadro regulatório e do apoio governamental; a dimensão tecnológica está associada a criação e difusão de conhecimento, estoque de capital humano e redes de criação de valor; por fim, a dimensão de mercado trata da demanda de novos produtos, formação de novos segmentos de mercado, mecanismos de financiamento para inovação e nas características da demanda.

Kantis, Federico e Garcia (2018) desenvolvedores do Index of Dynamic Entrepreneurship (IDE) propuseram três indicadores para representar as oportunidades empreendedoras: condições de demanda, estrutura empresarial e plataforma CTI.

As condições de demanda mensuram o tamanho e dinamismo do mercado. Condições cruciais para a criação e crescimento dos novos negócios. Essas condições influenciam o volume de empreendedores, por exemplo, durante recessões, o empreendedorismo motivado por oportunidade surge em resposta a falta de empregos de qualidade, por outro lado, em períodos de prosperidade econômica, em que há oferta de empregos de qualidade, os empreendedores normalmente são motivados por oportunidades. 
A estrutura empresarial se refere ao impacto que as demandas das empresas de grande porte exercem sobre os novos negócios, sobretudo aqueles orientados aos negócios (B2B). Uma estrutura empresarial dinâmica representa uma força motriz para a criação de novas empresas, pois os novos negócios podem atuar como fornecedores ou integrar a cadeia de valor de empresas de grande porte.

A plataforma CTI captura o valor do conhecimento científico e tecnológico para o empreendedorismo. Instituições de Ciência e Inovação (C\&T) e empresas privadas que realizam $\mathrm{P} \& \mathrm{D}$ podem comercializar o conhecimento, por meio da transferência de tecnologia para empresas existentes ou por meio da criação de spin-offs.

O modelo conceitual de Radosevic e Yoruk (2013) e o IDE apresentam e agregam mais medidas para capturar os aspectos multifacetados das oportunidades empreendedoras, enquanto o GEI se limita apenas ao uso de medidas sociais (GEM) e contextuais associadas a características institucionais/regulatórias (EFI). Dessa maneira, nessa pesquisa sugerimos a introdução de métricas mais amplas para captar a complexidade das oportunidades empreendedoras, isto é, os fatores que influenciam a capacidade dos indivíduos de reconhecer oportunidades, tanto de natureza institucional quanto tecnológica e mercadológica. Espera-se que a introdução dessas métricas reduzam as diferenças entre países desenvolvidos e em desenvolvimento.

\section{$5 \quad$ Considerações finais}

Nessa pesquisa, questionamos se os indicadores e subíndices do GEI são confiáveis e precisos para mensurar o empreendedorismo em nível de país.

No que se refere aos subíndices (constructos), calculamos os coeficientes de determinação, medida de ajustamento de um modelo linear geral em relação aos valores observados, como a regressão linear. Valores próximos a 1 são considerados significativos. Também calculamos a qualidade de ajuste do modelo por meio dos testes de validade preditiva $\left(\mathrm{Q}^{2}\right)$ e do tamanho do efeito $\left(\mathrm{F}^{2}\right)$. $\mathrm{O} \mathrm{F}^{2}$ determina quanto cada constructo é relevante para o ajuste do modelo e é obtido por meio de uma análise de sensibilidade dos constructos (inclusão e exclusão), valores maiores ou iguais a 0,35 são considerados grandes. $\mathrm{O} \mathrm{Q}^{2}$ avalia o quanto o modelo se aproxima do que se esperava dele, valores maiores que 0 são adequados. Quanto aos pilares, para identificar o quanto cada indicador se ajusta ao modelo, aplicamos o teste $t$ de Student, o qual, avalia as relações entre os indicadores e seus respectivos constructos.

Os valores para cada constructo obtidos por meio da avaliação dos coeficientes de determinação estão muito próximos a $1(\mathrm{ATT}=0,877$; $\mathrm{ABT}=0,927$; $\mathrm{ASP}=0,896)$, indicando que o modelo possui ajuste significativo. No que se refere ao teste de validade preditiva, obtivemos valores acima da média para cada constructo, o que significa que cada constructo é relevante para mensurar o fenômeno observado. Os constructos apresentaram desempenho semelhante quando foram submetidos ao teste do tamanho do efeito, onde, identificamos valores mais elevados que 0,35 para cada constructo, indicando que todos os 3 são úteis para o ajuste do modelo.

Os resultados para o coeficiente de determinação, validade preditiva e tamanho do efeito, indicam que cada constructo é relevante para o modelo. No que se refere aos indicadores que compõe cada constructo, somente o pilar opportunity perception apresentou valor $\mathrm{t}$ de Student inferior a 1,96 , fato que indica que o pilar não se ajusta ao modelo. Trata-se de uma 
medida combinada da percepção da população sobre as oportunidades empreendedoras e do papel do Estado na carga regulatória e diretos a propriedade. No entanto, apenas 34 dos 137 países avaliados pelo GEI $(24,82 \%$ da amostra) apresentam pontuação superior a 0,50 (a maioria deles, economias desenvolvidas) no pilar opportunity perception, fator que contribui para o não ajuste desse indicador ao modelo.

Ainda que o teste $t$ indique que o pilar não se ajusta ao modelo, podemos concluir, baseados nos demais resultados para os indicadores e subíndices que o GEI é um índice confiável e preciso para mensurar o empreendedorismo em nível de país. No entanto, o pilar opportunity perception deve ser reformulado, pois pode fornecer diagnósticos imprecisos aos formuladores de política, isto é, para melhorar o desempenho do EE nacional nesse pilar, é necessário promover o empreendedorismo, mas como o pilar não se ajustar ao modelo os esforços para melhorar o desempenho de um país podem ser prejudicados.

Por outro lado, as métricas agregadas no pilar opportunity perception podem não ser suficientemente adequadas para capturar os aspectos multifacetados das oportunidades empreendedoras, dessa maneira, agregar mais variáveis, especialmente aquelas associadas a tecnologia e ao mercado, podem fornecer novas perspectivas e ajustar o indicador ao modelo GEI.

Diante do exposto, recomendamos que os indicadores de empreendedorismo considerem os aspectos multifacetados do reconhecimento e exploração de oportunidades empreendedoras. Também consideramos, que estudos sobre EE em nível de país considerem as diferenças entre países.

\section{Referências bibliográficas}

ÁCS, Z. J.; AUDRETSCH, D. B.; LEHMANN, E. E. The knowledge spillover theory of entrepreneurship. Small Business Economics. v. 41, n. 1, p. 757-774, 2013

ÁCS, Z. J., et al. National systems of entrepreneurship. Small Business Economics. v. 46, n. 4, p. 527-535, 2016

ÁCS, Z. J.; AUTIO, E.; SZERB, L. National Systems of Entrepreneurship: Measurement issues and policy implications. Research Policy. v. 43, n. 3, p. 476-494, 2014

ÁCS, Z. J., et al. The knowledge spillover theory of entrepreneurship. Small Business Economics. v. 32, n. 1, p. 15-30, 2009

ÁCS, Z. J.; SZERB, L. The Global Entrepreneurship Index (GEINDEX). Foundations and Trends ${ }^{\circledR}$ in Entrepreneurship. v. 5, n. 5, p. 341-435, 2009

ÁCS, Z. J.; SZERB, L.; AUTIO, E. Global Entrepreneurship Index 2016. Washington, D.C.: The Global Entrepreneurship and Development Institute. 2016

ÁCS, Z. J., et al. The Regional Application of the Global Entrepreneurship and Development Index (GEDI): The Case of Spain. Regional Studies. v. 49, n. 12, p. 1977-1994, 2014

ÁCS, Z. J.; SZERB, L. A.; LLOYD, A. Global Entrepreneurship Index. Washington, D.C.: The Global Entrepreneurship and Development Institute. 2018

ALVEDALEN, J.; BOSCHMA, R. A critical review of entrepreneurial ecosystems research: towards a future research agenda. European Planning Studies. v. 25, n. 6, p. 887-903, 2017 ATIASE, V. Y.; MAHMOOD, S.; BOTCHIE, D. Developing entrepreneurship in Africa: investigating critical resource challenges. Journal of Small Business and Enterprise Development. v. 25, n. 4, p. 644-666, 2018 
AUDRETSCH, D. B., et al. Local entrepreneurship in context. Regional Studies. v. 46, n. 3, p. 379-389, 2012

AUTIO, E., et al. Entrepreneurial innovation: The importance of context. Research Policy. v. 43, n. 7, p. 1097-1108, 2014

BAUMOL, W. J. Entrepreneurship, management, and the structure of payoffs. London: MIT Press. 1993

CODURAS, A.; AUTIO, E. Comparing subjective and objective indicators to describe the national entrepreneurial context: the Global Entrepreneurship Monitor and the Global Competitiveness Index contributions. Investigaciones Regionales. v. 26, n., p. 47-74, 2013

COHEN, B. Sustainable valley entrepreneurial ecosystems. Business Strategy and the Environment. v. 15, n. 1, p. 1-14, 2006

FELD, B. Startup communities: Building an entrepreneurial ecosystem in your city. New Jersey: John Wiley \& Sons. 2012

GEM. Global entrepreneurship monitor 2017/2018 global report. London: L. B. S. GLOBAL ENTREPRENEURSHIP RESEARCH ASSOCIATION. 2018

GIRAUDO, E.; GIUDICI, G.; GRILLI, L. Entrepreneurship policy and the financing of young innovative companies: Evidence from the Italian Startup Act. Research Policy. v. 48, n. 9, p. $1-18,2019$

GUDERGAN, S. P., et al. Confirmatory tetrad analysis in PLS path modeling. Journal of Business Research. v. 61, n. 12, p. 1238-1249, 2008

HAIR, J. F., et al. A primer on partial least squares structural equation modeling (PLS-SEM). Los Angeles: SAGE. 2014

HAIR, J. F., et al. Primer on partial least squares structural. Los Angeles: SAGE Publications. 2016

HART, D. M. (Ed.) The emergence of entrepreneurship policy: Governance, start-ups, and growth in the U.S. Knowledge economy. Cambridge: Cambridge University Press. 2003

ISENBERG, D. How to start an entrepreneurial revolution. Harvard Business Review. v., n., p. $1-11,2010$

ISENBERG, D. (2011). The entrepreneurship ecosystem strategy as a new paradigm for economic policy principles for cultivating entrepreneurs. Presentation at the Institute of International and European Affairs, May 12, 2011. Dublin Ireland.: 13.

KANTIS, H.; FEDERICO, J.; GARCIA, S. I. Opening the blackbox of ecosystem diversity around the globe: The Index of Dynamic Entrepreneurship - IDE 2018. Rafaela: Associación Civil Red Pymes Mercosur. 2018

KUTNER, M. H., et al. Applied linear models. New York: McGraw-Hill Education. 2004

LAFUENTE, E.; SZERB, L.; ÁCS, Z. J. Country level efficiency and national systems of entrepreneurship: a data envelopment analysis approach. The Journal of Technology Transfer. v. 41, n. 6, p. 1260-1283, 2015

LINK, A. N.; SCOTT, J. T. Government as entrepreneur: Evaluating the commercialization success of SBIR projects. Research Policy. v. 39, n. 5, p. 589-601, 2010

MASON, C.; BROWN, R. Entrepreneurial ecosystems and growth-oriented enterprises. Paris: OCDE Publishing. 2014

MOORE, J. F. Predators and prey: A new ecology of competition. Harvard Business Review. v. 71, n., p. 75-86, 1993 
NECK, H. M., et al. An Entrepreneurial System View of New Venture Creation. Journal of Small Business Management. v. 42, n. 2, p. 190-208, 2004

OECD. Fostering entrepreneurship. Paris: OECD. 1998

QIAN, H.; ÁCS, Z. J. An absorptive capacity theory of knowledge spillover entrepreneurship.

Small Business Economics. v. 40, n. 2, p. 185-197, 2013

RADOSEVIC, S.; YORUK, E. Entrepreneurial propensity of innovation systems: Theory, methodology and evidence. Research Policy. v. 42, n. 5, p. 1015-1038, 2013

RIBEIRO-SORIANO, D.; MARTÍN-GALINDO, M.-Á. Government policies to support entrepreneurship. Entrepreneurship \& Regional Development. v. 24, n. 9-10, p. 861-864, 2012

RINGLE, C. M.; DA SILVA, D.; BIDO, D. D. S. Structural Equation Modeling with the Smartpls. Revista Brasileira de Marketing. v. 13, n. 02, p. 56-73, 2014

SHANE, S. Why encouraging more people to become entrepreneurs is bad public policy. Small Business Economics. v. 33, n. 2, p. 141-149, 2009

SMALLBONE, D. Entrepreneurship policy: Issues and challenges. Small Business Policy. v. 23, n. 3, p. 201-218, 2016

SPIGEL, B. The Relational Organization of Entrepreneurial Ecosystems. Entrepreneurship Theory and Practice. v. 41, n. 1, p. 49-72, 2017

SPILLING, O. R. The entrepreneurial system: On entrepreneurship in the context of a megaevent. Journal of Business Research. v. 23, n. 9, p. 1759-1769, 1996

STAM, E. Entrepreneurial Ecosystems and Regional Policy: A Sympathetic Critique. European Planning Studies. v. 23, n. 9, p. 1759-1769, 2015

STANGLER, D.; BELL-MASTERSON, J. Measuring an entrepreneurial ecosystem. Kansas City: Kauffman Foundation. 2015

SZERB, L. The Comparison of the Global Entrepreneurship Monitor and the Global Entrepreneurship and Development Index Methodologies. Foundations and Trends ${ }^{\circledR}$ in Entrepreneurship. v. 9, n. 1, p. 1-142, 2013

SZERB, L.; ÁCS, Z. J. The Global Entrepreneurship Index (GEINDEX). Foundations and Trends ${ }^{\circledR}$ in Entrepreneurship. v. 5, n. 5, p. 341-435, 2009

SZERB, L., et al. REDI: The Regional Entrepreneurship and Development Index - Measuring regional entrepreneurship. Luxembourg: Office of the European Union. 2014

SZERB, L.; TRUMBULL, W. N. Entrepreneurship development in Russia: is Russia a normal country? An empirical analysis. Journal of Small Business and Enterprise Development. v. 25, n. 6, p. 902-929, 2018

TASNIM, N.; AFZAL, M. N. I. An empirical investigation of country level efficiency and national systems of entrepreneurship using Data Envelopment Analysis (DEA) and the TOBIT model. Journal of Global Entrepreneurship Research. v. 8, n. 1, p., 2018

THU HA, N.; BA HOA, L. Evaluating entrepreneurship performance in Vietnam through the global entrepreneurship development index approach. Journal of Developmental Entrepreneurship. v. 23, n. 1, p. 644-666, 2018

VAN DE VEN, A. The development of an infrastructure for entrepreneurship. Journal of Business Venturing. v. 8, n., p. 211-230, 1993

WEF. Entrepreneurial ecosystems around the globe and company growth dynamics. Geneva: W. E. FORUM. 2013 